Prefitem

IP/BBSR/94-02

IMSC/94-02

January, 1994

\title{
Exact Duality and Nilpotent Gauging
}

\author{
Alok Kumar \\ Institute of Physics \\ Bhubaneswar-751005, India \\ and \\ SWAPNA MAHAPATra* \\ Institute of Mathematical Sciences \\ C. I. T. Campus, Madras-600113, India
}

\begin{abstract}
We obtain new duality transformations, relating some exact string backgrounds, by defining the nilpotent duality. We show that the ungauged $S L(2, R)$ WZW model transforms by its action into the three dimensional plane wave geometry. We also give the inverse transformation from the plane wave to the $S L(2, R)$ model and discuss the implications of the results.
\end{abstract}

^ e-mail: kumar@iopb.ernet.in, swapna@imsc.ernet.in 
The study of the classical solutions in string theory has received much attention in recent times. There have been two aspects of this study. The first one is to investigate the solutions of the low energy classical action [1] and the second one to find the exact solutions [2] [3] [4] [5]. The solutions of the second type are more interesting from the string point of view as they correspond to the two dimensional conformal field theories. The Wess-Zumino-Witten model is one of the examples of such a solvable conformal field theory. Many interesting solutions as exact conformal backgrounds have been obtained, the first one being the celebrated two dimensional black hole solution of Witten, which has been obtained as a gauged $S L(2, R)$ WZW model [2]. It was shown there that the conventional gauging (axial or vector) of a $U(1)$ subgroup of the $S L(2, R)$ WZW model leads to a two dimensional singular target space having the structure of a black hole solution. This has been generalized to higher dimensions also. For example, $\frac{S L(2, R) \otimes U(1)}{U(1)}$ theory corresponds to a three dimensional black string solution [4]. Many of these solutions are related by $O(d, d)$ transformations $[6-12]$.

For the $S L(2, R)$ theory, it is known that the axial and vector gauged models are related to each other via duality transformations [13] and are equivalent as conformal field theories though the target space geometry is very different in both the cases. The generalizations of these results to larger duality groups such as $O(d, d, Z)[14]$ have also been done. In these cases, the dual backgrounds were found corresponding to the isometry groups which were simply translations of some of the coordinates. Recently, the exact duality transformations have also been found for a number of cases [15] [16] [17]. The generalizations to the nonabelian isometry groups [18] have been considered as well.

In this paper, we investigate the duality with respect to the nilpotent subgroups. Earlier, it has been pointed out [3] that one can gauge the parabolic sub- 
group of $S L(2, R)$ in a consistent manner. This gives rise to the Liouville theory, where two of the degrees of the freedom are removed by a constraint and the resulting target space is one dimensional. This result has been confirmed through an explicit construction in [19], where a one dimensional target manifold was obtained by gauging the $S L(2, R)$ WZW model by its nilpotent subgroup $E(1)$.

Motivated by this, we have investigated the relationship between the duality transformations and the nilpotent gauging and show that the three dimensional plane wave solution can be related to the original $S L(2, R)$ WZW model by its action. It is interesting to note that both the initial and the final solutions in this case are exact string backgrounds. Duality transformations relating exact string backgrounds have been recently presented in another contexts [15] [16] [17]. For example in [15], plane wave solutions in arbitrary dimension were presented as duality invariant class. However, our duality transformations relate the solutions within the class of [15]to the ones outside.

The way one finds the dual background is the following: one first gauges the isometry and adds to the action a Lagrange multiplier term. The gauge fields are nondynamical in the theory in the sense that they do not have a kinetic term. If one integrates out the Lagrange multiplier term, one gets back the original model. On the other hand, by integrating by parts the Lagrange multiplier term and then integrating out the gauge fields, one obtains the dual action, in which the Lagrange multiplier is a new dynamical fields [20]. Similar techniques have been used for nonabelian duality also, but there the issues are somewhat more involved [18].

The plan of the paper is the following: we shall consider the nilpotent gauging of the $S L(2, R)$ WZW model, where we gauge the subgroup $E(1)$. We shall use the standard duality prescription to obtain the dual backgrounds for both the 
axial and the vector gauged action. Interestingly, we find that the dual geometry corresponding to the $S L(2, R)$ WZW model is a three dimensional target space, representing a plane wave solution. Such plane wave solutions have been discussed recently by various people in the context of WZW models based on non-semisimple groups [21]. The dual metric we have obtained has two isometries and a covariantly constant null killing vector. We then apply the duality inversion with respect to these two isometries and obtain the original ungauged WZW model for one of these.

As will be discussed in the end, our duality transformations do not belong to any of the class of transformations, approximate or exact, presented earlier [15] [16] [17]. The reason being the appropriate use of the null isometry, as suggested in [15], for generating new backgrounds.

We now start by considering the WZW model based on the group $G=S L(2, R)$. We parametrize the group manifold by,

$$
g=\left(\begin{array}{cc}
a & u \\
-v & b
\end{array}\right), \quad \text { with } \quad a b+u v=1
$$

The group action for the axial gauging is given by $g \rightarrow h g h$, where $g \in G$, $h \in H$ and $H$ is the abelian subgroup of $G$. On the other hand, the group action for the vector gauging is given by, $g \rightarrow h g h^{-1}$. Here, we shall consider the gauging of the nilpotent subgroup $E(1)$ of $S L(2, R)$, where the subgroup is generated by $\sigma^{+}=\sigma_{3}+i \sigma_{2}$. The term nilpotent reflects the fact that $\left(\sigma^{+}\right)^{2}=0$. Let us first consider the axial gauged model. The gauged action in this case can be written as,

$$
S_{a}(g, A)=S(g)+\frac{k}{2 \pi} \int d^{2} z \operatorname{Tr}\left(\bar{A} g^{-1} \partial g+A \bar{\partial} g g^{-1}+A \bar{A}+g^{-1} A g \bar{A}\right)
$$


where, $S(g)$ is the ungauged $S L(2, R)$ WZW model action, which is given by,

$$
S(g)=\frac{k}{4 \pi} \int_{\Sigma} d^{2} z \operatorname{Tr}\left(g^{-1} \partial g g^{-1} \bar{\partial} g\right)-\frac{k}{12 \pi} \int_{B} \operatorname{Tr}\left(g^{-1} d g \wedge g^{-1} d g \wedge g^{-1} d g\right)
$$

$B$ is a three manifold, whose boundary is $\Sigma$. The gauge fields $A$ and $\bar{A}$ take values in the algebra of $H$ and they transform as,

$$
A \rightarrow h(A+\partial) h^{-1}, \quad \bar{A} \rightarrow h^{-1}(\bar{A}+\bar{\partial}) h
$$

In terms of the above parametrization, the ungauged WZW model action becomes,

$$
S(g)=-\frac{k}{4 \pi} \int d^{2} z(\partial u \bar{\partial} v+\bar{\partial} u \partial v+\partial a \bar{\partial} b+\bar{\partial} a \partial b)+\frac{k}{2 \pi} \int d^{2} z \log u(\partial a \bar{\partial} b-\bar{\partial} a \partial b)
$$

If $\epsilon$ is an infinitesimal gauge transformation parameter, then the above local symmetry is generated by,

$$
\begin{aligned}
\delta g & =\epsilon g+g \epsilon ; \\
\delta A_{i} & =-\partial_{i} \epsilon .
\end{aligned}
$$

We now gauge,

$$
\begin{aligned}
& \delta a=\epsilon(2 a-u-v) ; \\
& \delta b=\epsilon(-2 b-u-v) \\
& \delta u=\epsilon(a+b) \\
& \delta v=\epsilon(a+b) .
\end{aligned}
$$

The gauge invariant parameters for this case are $x=u-v$ and $w=a-b-u-v$ 
and the complete gauged action can be written as,

$$
\begin{aligned}
S(g, A) & =S(g)+\frac{k}{2 \pi} \int d^{2} z[\bar{A}(b \partial a+u \partial v+v \partial a-a \partial v-v \partial u-a \partial b-b \partial u+u \partial b) \\
& +A(b \bar{\partial} a+v \bar{\partial} u-b \bar{\partial} v+v \bar{\partial} b+u \bar{\partial} a-a \bar{\partial} u-u \bar{\partial} v-a \bar{\partial} b) \\
& \left.+A \bar{A}(-a+b+u+v)^{2}\right] .
\end{aligned}
$$

We fix the gauge by choosing $a+b=0$. So the gauge fixed action has the form,

$$
\begin{aligned}
S(g, A) & =-\frac{k}{4 \pi} \int d^{2} z(\partial u \bar{\partial} v+\bar{\partial} u \partial v-2 \partial a \bar{\partial} a) \\
& +\frac{k}{2 \pi} \int d^{2} z\left[-\frac{1}{2} \bar{A}(x \partial w-w \partial x)+\frac{1}{2} A(x \bar{\partial} w-w \bar{\partial} x)-w^{2} A \bar{A}\right]
\end{aligned}
$$

After integrating out the gauge fields one obtains,

$$
S=\frac{k}{2 \pi} \int d^{2} z\left[\frac{\partial w \bar{\partial} w}{w^{2}}\right]
$$

The background metric, antisymmetric tensor and the dilaton fields corresponding to the action (10)can be written as

$$
\begin{aligned}
d s^{2} & =\frac{k}{2} \frac{(d w)^{2}}{w^{2}}, \\
B & =0
\end{aligned}
$$

and

$$
\Phi=-\log w+\text { constant }
$$

This is just the $S L(2, R) / E(1)$ gauged WZW model [19]. The resulting target space is one dimensional and the effective action corresponds to the Liouville action. 
Now we obtain the model dual to the $S L(2, R)$ WZW model with respect to its nilpotent subgroup. For this, we start with the original $S L(2, R)$ WZW action, gauge it and then add the Lagrange multiplier term to the action. The gauged action with the Lagrange multiplier term (which is gauge invariant) is given by,

$$
S(g, A, \lambda)=S(g)+\frac{k}{2 \pi} \int d^{2} z\left[\bar{A} J+A \bar{J}-w^{2} A \bar{A}\right]+\frac{k}{2 \pi} \int d^{2} z(A \bar{\partial} \lambda-\bar{A} \partial \lambda)
$$

where the currents $J$ and $\bar{J}$ are given by,

$$
\begin{aligned}
& J=[b \partial a+u \partial v+v \partial a-a \partial v-v \partial u-a \partial b-b \partial u+u \partial b] ; \\
& \bar{J}=[b \bar{\partial} a+v \bar{\partial} u-b \bar{\partial} v+v \bar{\partial} b+u \bar{\partial} a-a \bar{\partial} u-u \bar{\partial} v-a \bar{\partial} b]
\end{aligned}
$$

and $\lambda$ is the Lagrange multiplier. The gauge fixed action is obtained by putting the condition $a+b=0$. Now fixing the gauge and integrating out the gauge fields, the dual sigma model action is obtained in terms of the gauge invariant parameters and $\lambda$. The expression is given by,

$$
S=\frac{k}{2 \pi} \int d^{2} z\left[\frac{\partial w \bar{\partial} w}{w^{2}}-\frac{\partial \lambda \bar{\partial} \lambda}{w^{2}}-\frac{1}{2} \frac{\partial \lambda}{w^{2}}(x \bar{\partial} w-w \bar{\partial} x)+\frac{1}{2} \frac{\bar{\partial} \lambda}{w^{2}}(x \partial w-w \partial x)\right]
$$

where we have used the values of $A$ and $\bar{A}$ as (obtained by using their equation of motion),

$$
\begin{aligned}
& A=-\frac{1}{w^{2}}\left[\partial \lambda+\frac{1}{2}(x \partial w-w \partial x)\right] ; \\
& \bar{A}=\frac{1}{w^{2}}\left[\partial \lambda+\frac{1}{2}(x \bar{\partial} w-w \bar{\partial} x)\right) .
\end{aligned}
$$

From the above expression, we can read off the values for the dual background 
metric $\tilde{g}_{i j}$ and antisymmetric tensor field $\tilde{b}_{i j}$. The dual metric is given by,

$$
d \tilde{s}^{2}=\frac{k}{2 w^{2}}\left[(d w)^{2}-(d \lambda)^{2}-x d \lambda d w+w d \lambda d x\right]
$$

The dual dilaton is found to be,

$$
\tilde{\Phi}=-\log w+\text { constant }
$$

and $\tilde{b}_{i j}$ is zero. Unlike the $\frac{S L(2, R)}{E(1)}$ model, one extra degree of freedom does not get removed and the target space is three dimensional. The only non zero component of the Ricci tensor is $R_{22}=-\frac{2}{w^{2}}$. The curvature scalar $R$ is zero as $g^{22}$ is zero (here $w \equiv 1, \lambda \equiv 2$ and $x \equiv 3$ ). These backgrounds satisfy the one loop beta function equations namely,

$$
\begin{array}{r}
R_{\mu \nu}+2 \nabla_{\mu} \nabla_{\nu} \tilde{\Phi}=0 \\
R+4 / k^{\prime}-4(\nabla \tilde{\Phi})^{2}+4 \nabla^{2} \tilde{\Phi}=0
\end{array}
$$

We have also explicitly verified that they satisfy the two loop beta function equations.

Recently, in a series of papers [22], the path integral formulations of the gauged WZW model has been given to compute the exact string backgrounds. It was found that the quantum action relevant for computing the exact backgrounds differs from the classical one by a term which is proportional to $\operatorname{Tr}[A \bar{A}]$. In our case this term is zero due to the nilpotency of the gauged subgroup. As a result we expect the backgrounds in eqns. (17)-(18)to be exact to all orders in sigma model. 
Also, by making a coordinate redefinition,

$$
y=\frac{x}{w}
$$

which is well defined, since $w \neq 0$, we can rewrite the dual metric as,

$$
d \tilde{s}^{2}=\frac{k}{2}\left[\frac{1}{w^{2}}\left((d w)^{2}-(d \lambda)^{2}\right)+d \lambda d y\right]
$$

The metric $\tilde{g}_{i j}$ now is independent of $\lambda$ and $y$. After shifting $y \rightarrow 2 y$, we obtain,

$$
d \tilde{s}^{2}=\frac{k}{2}\left[\frac{1}{w^{2}}\left((d w)^{2}-(d \lambda)^{2}\right)+2 d \lambda d y\right]
$$

which corresponds to a plane wave solution. The killing vector (in $\lambda$-direction) $k_{a}=(0,1,0)$ is a null vector as $k_{a} k^{a}=0$ and $k_{a ; n}=k_{a, n}-\Gamma_{a n}^{\lambda} k_{\lambda}=0$. This implies that $k_{a}$ is a covariantly constant null killing vector. The existence of such a vector and the form of the metric implies that the dual background is basically a three dimensional plane wave solution, which is known to be an exact solution of string theory to all orders [23]. This is consistent with the arguments presented above.

We have also analyzed the vector gauged dual model. The vector gauged action is given by,

$$
S_{v}(g, A)=S(g)+\frac{k}{2 \pi} \int d^{2} z \operatorname{Tr}\left(A \bar{\partial} g g^{-1}-\bar{A} g^{-1} \bar{\partial} g+A \bar{A}-g^{-1} A g \bar{A}\right) .
$$

The vector gauge transformation is given by, $g \rightarrow h g h^{-1}$. The local vector symmetry is generated by, $\delta g=g \epsilon-\epsilon g$. We gauge the symmetry,

$$
\begin{aligned}
& \delta a=\epsilon(v-u) \\
& \delta b=\epsilon(u-v) ; \\
& \delta u=\epsilon(a-b-2 u) ; \\
& \delta v=\epsilon(-a+b+2 v) .
\end{aligned}
$$

Here the gauge invariant parameters are $x^{\prime}=a+b$ and $w=a-b-u-v$. We 
choose the gauge $u-v=0$ and again add the Lagrange multiplier term to the gauged action. The gauge fixed action is given by,

$$
\begin{aligned}
S(g, A, \lambda) & =-\frac{k}{4 \pi} \int d^{2} z(2 \partial u \bar{\partial} u+\partial a \bar{\partial} b+\bar{\partial} a \partial b) \\
& +\frac{k}{2 \pi} \int d^{2} z\left[-\frac{1}{2} A\left(x^{\prime} \bar{\partial} w-w \bar{\partial} x^{\prime}\right)-\frac{1}{2} \bar{A}\left(x^{\prime} \partial w-w \partial x^{\prime}\right)+w^{2} A \bar{A}\right] \\
& +\frac{k}{2 \pi} \int d^{2} z(A \bar{\partial} \lambda-\bar{A} \partial \lambda)
\end{aligned}
$$

Integrating out the gauge fields as before we obtain,

$$
S=\frac{k}{2 \pi} \int d^{2} z\left[\frac{\bar{\partial} w \partial w}{w^{2}}+\frac{\bar{\partial} \lambda \partial \lambda}{w^{2}}+\frac{x^{\prime}}{2 w^{2}}(\partial \lambda \bar{\partial} w+\bar{\partial} \lambda \partial w)-\frac{1}{2 w}\left(\partial \lambda \bar{\partial} x^{\prime}+\bar{\partial} \lambda \partial x^{\prime}\right)\right]
$$

From the above expression, we find that the dual antisymmetric tensor field is again zero and the dual metric is given by,

$$
d \tilde{s}^{2}=\frac{k}{2}\left[\frac{1}{w^{2}}\left((d w)^{2}+(d \lambda)^{2}\right)+\frac{x^{\prime}}{w^{2}} d \lambda d w-\frac{1}{w} d \lambda d x^{\prime}\right]
$$

The dual dilaton is again given by,

$$
\tilde{\Phi}=-\log w+\text { constant }
$$

As in the case of axial gauging case, we define, $y=\frac{x^{\prime}}{w}$ and shifting $y \rightarrow 2 y$ we obtain,

$$
d \tilde{s}^{2}=\frac{k}{2}\left[\frac{1}{w^{2}}\left((d w)^{2}+(d \lambda)^{2}\right)-2 d \lambda d y\right]
$$

This is again a plane wave solution in three dimensions. We note that the metric in the two different gauged models, eqns.(22)and (29), are esentially same upto analytic continuations. This follows from the fact that $A \bar{A}$ term is zero in 
eqns.(2) and (23). As a result, the gauged actions are related by a field redefinition $\bar{A} \rightarrow-\bar{A}$. Taking this into account the dual metric in two different gauging, after a shift $\lambda \rightarrow \lambda-y$, become (upto analytic continuation),

$$
d \tilde{s}^{2}=\frac{k}{2}\left[\frac{1}{w^{2}}\left((d w)^{2}-(d \lambda)^{2}\right)-(d y)^{2}\left(\frac{1+2 w^{2}}{w^{2}}\right)+2 d \lambda d y\left(\frac{1+w^{2}}{w^{2}}\right)\right]
$$

Now, if we apply the duality inversion [6] [20]with respect to the isometry in $y$ direction for the metric (30), we get,

$$
\begin{aligned}
d \tilde{s}^{2} & =\frac{k}{2}\left[\frac{1}{w^{2}}(d w)^{2}-\left(\frac{w^{2}}{1+2 w^{2}}\right)(d y)^{2}+\left(\frac{w^{2}}{1+2 w^{2}}\right)(d \lambda)^{2}\right] \\
\tilde{B}^{\prime} & =-1 ; \\
\tilde{\Phi}^{\prime} & =2 \phi+\text { constant } .
\end{aligned}
$$

More interesting is the case of $\lambda$ isometry for the metric (30)for which

$$
\begin{aligned}
d \tilde{s}^{2} & =\frac{k}{2}\left[\frac{1}{w^{2}}(d w)^{2}-w^{2}(d \lambda)^{2}+w^{2}(d y)^{2}\right] \\
\tilde{B}_{\lambda y}^{\prime} & =-\left(1+w^{2}\right) \\
\tilde{\Phi}^{\prime} & =\text { constant } .
\end{aligned}
$$

Interestingly the final background in (32)is same as the ungauged $S L(2, R)$ WZW model. To show this we first find, by an explicit computation, that the Ricci tensors $R_{\mu \nu}$ for the metric in eqns.(32)is proportional to $g_{\mu \nu}$. As a result this is the metric for a maximal symmetric space. More explicitly, by coordinate reparametrizations and analytic continuations, the metric in eqn.(32)can be trans- 
formed to the Robertson-Walker form in three dimensions:

$$
d \tilde{s}^{\prime 2}=\frac{k}{2}\left[(d t)^{2}+\exp (2 t)\left[(d r)^{2}+r^{2}(d \theta)^{2}\right]\right]
$$

By another coordinate transformation, similar to the one in four dimensional case [24], this can be written in the static form:

$$
d \tilde{s}^{2}=\frac{k}{2}\left[\left(1-r^{2}\right)(d t)^{2}+\left(1-r^{2}\right)^{-1}(d r)^{2}+r^{2}(d \theta)^{2}\right]
$$

which, after some analytic continuations, can be identified with the metric of the three dimensional black hole [25] [26]. Together with the antisymmetric tensor and the dilaton as in eqn.(32), it can be interpreted as the ungauged $\mathrm{SL}(2, \mathrm{R})$ WZW model [26].

To conclude, in this paper we have presented the duality transformations which relate two exact solutions of string theory, namely the WZW models and the plane wave sulutions. We would like to point out once again that the duality transformations applied in our case are outside the set of $O(2,2)$ transformations [10] [16] that are normally considered for a one coordinate dependent background in three spacetime dimensions. For generating inequivalent backgrounds, the $O(2,2)$ transformations have only one nontrivial parameter [8]. One can use this to transform away the antisymmetric tensor present in the original ungauged WZW model. However, it turns out that this demand fixes the background for other fields as well. The final model in this case is a product space of the 2-d black hole with a flat coordinate [10] [16]. In the present case we once again have a vanishing antisymmetric tensor. But the other backgrounds are different than the product space just discussed. Hence the plane wave solution does not belong to the class of models 
related to the ungauged $\mathrm{SL}(2, \mathrm{R})$ model by $O(2,2)$ transformations. A more likely interpretation of our transformations is probably in terms of a suggestion presented in [15] where it was pointed put that the duality transformation, after mixing the null isometry with the other ones, can give rise to new consistent backgrounds. It will be interesting to examine this proposal further. It will also be interesting to examine these results for the higher dimensional plane wave solutions and WZW models as well.

Acknowledgement: One of us (S. M.) would like to thank Institute of Physics, Bhubaneswar for its hospitality, where part of this work was done.

\section{REFERENCES}

N1 G. Gibbons and K. Maeda, Nucl. Phys. B298 (1988) 741; D. Garfinkle, G. Horowitz and A. Strominger, Phys. Rev. D43 (1991) 3140; M. Mueller, Nucl. Phys. B337 (1990) 37; G. Mandal, A. M. Sengupta and S. Wadia, Mod. Phys. Lett. A6 (1991) 1685. N2 E. Witten Phys. Rev. D44 (1991) 314; N3 R. Dijkgraaf, H. Verlinde and E. Verlinde, Nucl. Phys. B 271(1992)269. N4 J. Horne and G. Horowitz, Nucl. Phys. B368(1992) 444. N5 S. Kar and A. Kumar, Phys. Lett.

B 291(1992)246; S. Mahapatra, Mod. Phys. Lett. A7(1992)2999; S. Kar, S. P. Khastgir and G. Sengupta, Phys. Rev. D47(1993)3643. N6 T. Busher, Phys. Lett. B201 (1988) 466; ibid B194 (1987) 59. N7 G. Veneziano, Phys. Lett. B265 (1991) 287; K. Meissner and G. Veneziano, Phys. Lett. B267 (1991) 33; M. Gasperini, J. Maharana and G. Veneziano, Phys. Lett. B272 (1991) 277. N8 A. Sen, Phys. Lett. B271 (1991) 295; ibid B274 (1991) 34; S. F. Hassan and A. Sen, Nucl. Phys. B375 (1992) 103. N9 S. Khastgir and A. Kumar, Mod. Phys. Lett. A6 (1991) 3365; S. Khastgir and J. Maharana, Class. Quant. Grav. (1993). N10 A. Kumar, Phys. Lett. 
B293 (1992) 49. N11 J. Maharana and J. Schwarz, CALT-68-1790. N12 J. Horne, G. Horowitz and A. Steif, Phys. Rev. Lett. 68 (1992) 568. N13 E. Kiritsis, Mod. Phys. Lett. A6 (1991) 2871. N14 A. Giveon and M. Rocek, Nucl. Phys. B380 (1992) 128. N15 C. Klimcik and A. Tseytlin, CERN-TH.7069/93 (hepth/9311012). N16 D. Gershon, TAUP-2121-93, (hepth/9311122). N17 E. Bergshoeff, I. Entrop and R. Kallosh, SU-ITP-93-37, UG-8/93 (hepth/9401025). N18 X. de la Ossa and F. Quevedo, Nucl. Phys. B403 (1993) 377; A. Giveon and M. Rocek, ITPSB-93-44, RI-152-93 (hepth/9308154), E. Alvarez, L. Alvarez-Gaume, J. L. F. Barbon, Y. Lozano, CERN-TH.6991/93, FTUAM.93/28; M. Gasperini, R. Ricci and G. Veneziano, CERN-TH.6960/93, ROM2F/93/24 (hepth/9308112). N19 M. Alimohammadi, F. Ardalan and H. Arfaei, BONN-HE-93-12; SUTDP-93/72/3; IPM-93-007 (hepth/9304024). N20 M. Rocek and E. Verlinde, Nucl. Phys. B373 (1992) 630. N21 C. Nappi and E. Witten, IASSNS-HEP-93/61 (hepth/9310112); K. Sfetsos, THU-93-30 (hepth/9311010), THU-93-31 (hepth/9311093); E. Kiritsis and C. Kounnas, CERN-TH.7059/93 (hepth/9310202); D. I. Olive, E. Rabinovici and A. Schwimmer, SWA/93-94/15, WIS-93/1-CS, RI-93/69. N22 I. Bars and K. Sfetsos, Phys. Rev. D48 (1993) 844; A. Tseytlin, CERN preprint, hepth/9301015; K. Sfetsos, USC-93/HEP-S1 (hepth/9305074); K. Sfetsos and A. Tseytlin, CERNTH.6962/93, (hepth/9308018). N23 D. Amati and C. Klimcik, Phys. Lett. B219 (1989) 443; G. Horowitz and Steif, Phys. Rev. Lett. 64 (1990) 260; Phys. Rev. D42 (1990) 1950. N24 N. Birrell and P. Davies, Cambridge University Press (1982). N25 M. Banados, C. Teitelboim and J. Zanelli, Phys. Rev. Lett. 69 (1992) 1849. N26 A. Ali and A. Kumar, Mod. Phys. Lett. A8 (1993) 2045; G. Horowitz and Welch, Phys. Rev. Lett. 71 (1993) 328. 